\title{
Editorial
}

\section{What is the impact of systolic blood pressure lowering on preventing cardiovascular events?}

\author{
Journal of Human Hypertension \\ (2004) 18, S1. doi:10.1038/ \\ sj.jhh.1001794
}

The most important epidemiological fact of the late 1990s-the recognition that systolic blood pressure is the primary indicator of cardiovascular risk-is now having major clinical consequences. These proceedings explore this fascinating turnabout in clinical hypertension.

First, H Black reviews the foundations of the paradigm shift from the primacy of diastolic blood pressure (the only measure of risk used in the early trials of hypertension therapy) to the current recognition that systolic levels become the principal indicator of risk after the age of 50 years.

For a long time, the attention of the medical community was directed towards diastolic hypertension, the only measure of risk used in the early trials of hypertension therapy. Finally, in 1971, the Framingham Heart Study clearly showed that it was systolic blood pressure that more accurately described the risk of all the complications we attribute to hypertension. It was 22 years before JNC V also used systolic blood pressure to define hypertension. Since then the foundations of the paradigm have been dramatically shifted. In the American and European guidelines that followed for the management of arterial hypertension, systolic blood pressure became the primary focus of risk stratification and treatment goals.

Over the past few years, numerous randomized controlled trials have examined the treatment of patients with hypertension. Overall, these trials have shown that therapy, usually involving low doses of a diuretic, provides protection against both stroke and coronary disease even if the systolic blood pressure is not reduced to the usual goal of $140 \mathrm{mmHg}$.

$\mathrm{G}$ London provides evidence for the efficacy of indapamide $1.5 \mathrm{mg}$, sustained release (Indapamide $1.5 \mathrm{mg}$, sustained release brand names: Natrilix SR, Natrilix LP, Natrilix Retard, Natrilix AP, Fludex LP, Fludex retard, Fludex SR, Fludex $1.5 \mathrm{mg}$, Tertensif SR, Tertensif retard, Arifon retard, Pretanix), in lowering systolic blood pressure and thereby providing excellent cardiovascular and renal protection.

A recent study, with candesartan and amlodipine as comparators, confirms again that indapamide $1.5 \mathrm{mg}$, sustained release, a low-dose diuretic antihypertensive, is at least as effective as the major antihypertensive agents, particularly in reducing systolic blood pressure in all hypertensive patients including those with isolated systolic hypertension (ISH); in this ISH population, the benefit of indapamide $1.5 \mathrm{mg}$, sustained release, is increased by its remarkable effect on pulse pressure.

F Forette and co-workers provide a comprehensive review of the multiple randomized controlled trials performed over the past 30 years which have provided conclusive evidence of the particularly important protection against major cardiovascular events including stroke, heart failure and coronary heart disease by the use of diuretic-based therapy in elderly hypertensive patients.

$\mathrm{P}$ Verdecchia follows with a careful analysis of the relative role of reduction in systolic pressure per se in the regression of left ventricular hypertrophy that has been repeatedly observed in therapeutic trials.
Indapamide $1.5 \mathrm{mg}$, sustained release, has been found to provide excellent cardiovascular and renal protection in all types of hypertensive patients, notably in highrisk patients with left ventricular hypertrophy. The relative role played by the reduction in systolic pressure per se in the regression of left ventricular hypertrophy has been repeatedly observed in therapeutic trials. In the Left ventricular hypertrophy: Indapamide vs Enalapril (LIVE) study, indapamide $1.5 \mathrm{mg}$, sustained release, has been shown to decrease left ventricular hypertrophy significantly more than enalapril with the same decrease in systolic blood pressure. It seems likely that, beyond a blood pressure effect, indapamide $1.5 \mathrm{mg}$, sustained release, has a specific action on the cardiac myocytes.

The last paper in these proceedings summarizes the overall experiences with diuretics, particularly in low doses, for the treatment of hypertension and in the prevention of cardiovascular diseases.

The sponsor of these proceedings, Servier, is to be congratulated for providing this platform for a useful examination of the critical role of systolic hypertension. It is now obvious that systolic hypertension poses the greatest cardiovascular risk for the largest and fastest growing part of the hypertensive population, those over the age of 55 years. The evidence reviewed in these proceedings clearly documents the ability of diuretic-based therapy to protect these vulnerable patients.

NM Kaplan

The University of Texas

Southwestern Medical Center,

Dallas, TX, USA 